\title{
Vertex operator formulation of scattering around black-hole
}

\author{
I.Y. Park \\ Kavli Institute for Theoretical Physics, \\ Santa Barbara, California, USA \\ Center for Quantum Spacetime, Sogang University \\ Shinsu-dong 1, Mapo-gu, 121-742, South Korea \\ and \\ Division of Physical and Natural Sciences ${ }^{1}$, Philander Smith College \\ Little Rock, AR 72202, USA \\ inyongpark05@gmail.com
}

\begin{abstract}
We propose a full-fledged open string framework that seems suited to study the black hole information paradox. We set up a configuration to compute the scattering amplitude of a IIB open string around a D5-brane. The D5-brane is situated at the origin of a transverse D3-brane. A string perturbation theory is employed where the geometry of the D5-brane is treated as a potential. We reason that the setup is capable of reconciling the unitary evolution of states and information loss that is measured by an observer on the D3 brane. With the configurations of these kinds, the information loss is an apparent phenomenon: it is just a manifestation of the fact that the D3-observer does not have access to the "hair" of the D5 black brane.
\end{abstract}

\footnotetext{
${ }^{1}$ Home instutution
} 
Black holes have played important roles in recent developments in string theory $[1,2]$. They appear as relatively simple solutions of various supergravity theories whose horizon areas yield Bekenstein-Hawking entropies. (See, for example $[3,4,5,6]$.) The microscopic origin of the entropy was then understood $[1,2,7,8]$ by making a connection with D-branes $[9,10,11,12]$. One well-known puzzle in black hole physics is the black hole information paradox. Relatively recent discussions on the topic can be found in $[13,14,15,16,17]$. Since string theory is capable of describing gravitation, it should provide a proper setup to tackle the problem. There have also been studies on absorption cross sections of various black holes, e.g., [18, 19, 20, 21]. Most of these works are based on the low energy effective actions of string theory, supergravity or gauge/DBI theory. What is desired is a full-fledged vertex operator formulation that is designed, with a "realistic" touch, to compute various scattering amplitudes around a black hole. In this paper, we propose one such setup.

The system that we consider consists of a D5-brane and a complementary D3 brane. As shown in the figure below, they are entirely perpendicular. The scattering open strings move on the D3 brane. It also hosts an observer, therefore we may call it a "brane-world". To the observer, the D5 brane appears as a hole instead of a brane. Namely, confined within the brane, the observer only sees a "dot", the black hole. The scattering around the black hole is measured. A type IIB supergravity solution that corresponds to such a configuration have not been established in the literature although we expect its existence. The configuration might be obtained from a known solution. We briefly comment on this later. Intuitively it is obvious that the D5-brane provides an "escape passageway" for some of the incoming strings. This will cause a loss in the flux that is measured by the D3-observer, which will be interpreted as information loss. With the setup under consideration (and the similar kinds), it is clear that the loss is an apparent phenomenon: it is a simple reflection of the D3 observer's inaccessibility to the "hair" of the D5 brane. One of the goals of this paper is to provide a quantitative description of the scattering.

The picture can be put on computational ground with the following philosophy. What we propose as part of the setup is two-sided concerning how to deal with each of the geometries, the geometry due to D5 and that of the D3. It is based on our view that the two geometries should have different interpretations and therefore play different roles. In the spirit of previous works $[22,23,24,25]$ we attribute the geometry of D5-brane to the loop effects of the open strings that live in the D5-brane. Such physics is only indirectly visible, and only in a limited manner, to an observer who lives on the D3. Most of the detailed physics will be hidden: we propose to treat the geometry of the D5 brane as a potential that is felt by the scattering open strings. What it means in terms of the action is that we start with a non-linear sigma model [26, 27, 28] of D5brane geometry and view it as consisting of free pieces plus the potential terms. The potential will then affect the dynamics of the open strings moving on the D3. It is the part of the proposal that makes the setup different from those in the other literature. The second ingredient-which is also in the spirit of the previous works- is that we 
associate the geometry of the D3 brane with the loop effects ${ }^{1}$ of the scattering open string on the D3-brane. It implies, in the case where one considers only the tree level scattering (as we do in this work), that one may disregard the curved geometry due to the D3-brane: it will only play a role as counter terms in loop scattering diagrams.

The discussion so far leads to the following overall picture: we consider an open string moving on the D3-brane whose vertex operator has been constructed in [22] following [31]. As in the standard quantum mechanical formulation of scattering, we consider a two-point amplitude. The effect of the presence of a black hole in general is then introduced as a vertex operator insertion in the correlation function. The precise form of the potential is determined based on the two-dimensional non-linear sigma model of the D5-brane. The correlator can be evaluated by following the method in the previous works. Towards the end, we note that, in a full string description, genuine information loss does not occur in a generic configuration of the present kind. ${ }^{2}$

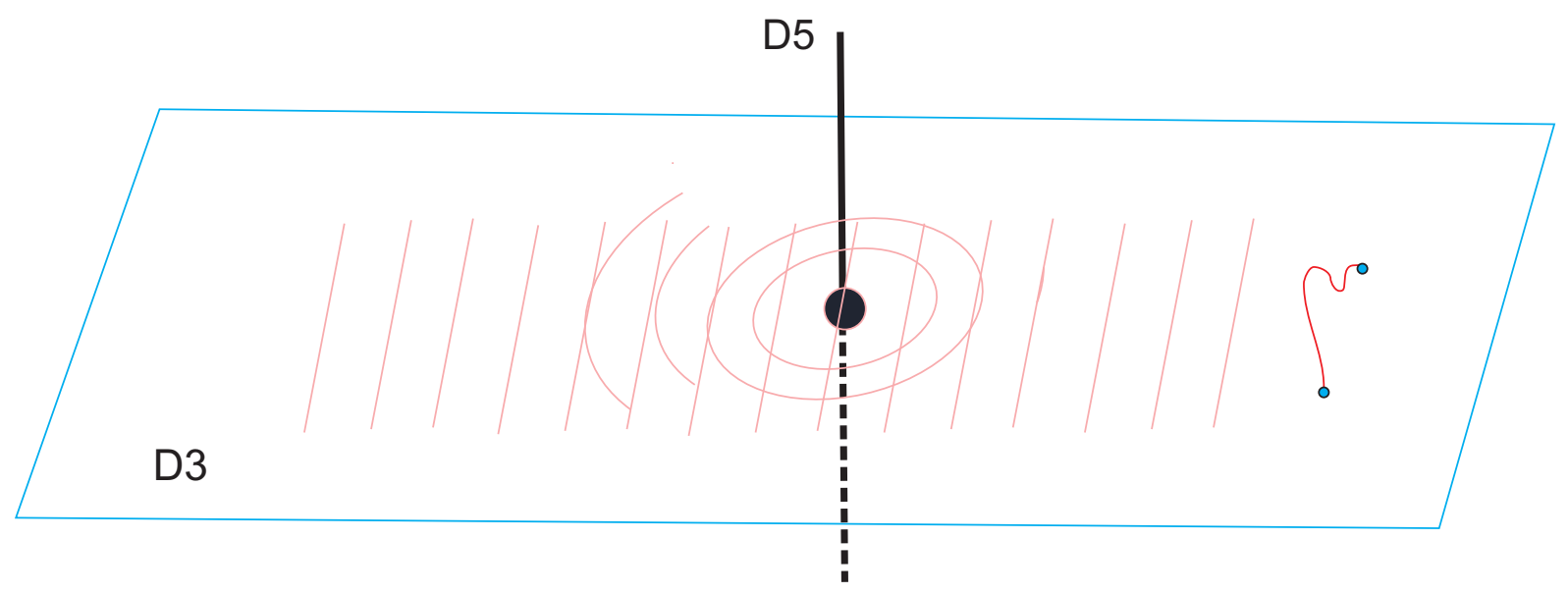

Figure 1: An open string scattering around a D5-brane

The detailed computation will be taken below but it may be worth thinking about what one should expect as a result of the computation. In order to answer the question, we try to develop a further intuition on the system. The first issue that we would like to address is a potential obstacle: whether we have a necessary tool for the computation. In general, studying physics near a black hole may require a non-perturbative tool that is unavailable. However, since we are interested mainly in the flux loss, we can adopt a procedure that is similar to the one developed in [24]. In this procedure, we consider

\footnotetext{
${ }^{1}$ The connection between loop effects and geometry goes back to the Fischler-Susskind mechanism [29]. The connection between open string loop effects and the D-brane geometry in the current context has been established at one-loop in [24] and partially established at two-loop in [25]. Work is in progress [30] for the three-loop order.

${ }^{2}$ There is a difference between an open string and a closed string. We will comment on this as well.
} 
what we call a large $R_{0}$-expansion where one expands the spatial D3-brane coordinate as

$$
\mathrm{X}^{u}=<X^{u}>+X^{u}=X_{0}^{u}+X^{u}, \quad R_{0}^{2}=\sum_{u}\left(X_{0}^{u}\right)^{2}
$$

Invoking Ehrenfest theorem, we interpret $R_{0}$ as the average location or the location in the classical sense of the string. ${ }^{3}$ In other words, by considering the large- $R_{0}$ expansion we are analyzing the dynamics of a string which has an average location at $R_{0}{ }^{4}$

We are ready to discuss what to expect as an outcome of the computation. For that purpose, we use an analogy with the quantum mechanical formulation of scattering. To avoid unnecessary complications, we consider non-relativistic scattering. The discussion will be heuristic.

If one sets out to analyze non-relativistic scattering around the D5-brane, one will consider a wave-function which schematically takes the form of

$$
e^{i k \cdot x}+\frac{e^{i k r}}{r^{n}} f\left(k^{\prime}, k\right)+\beta(\mathbf{r}) \psi_{D 5}
$$

The first two terms are standard except that the power of $\frac{1}{r}$ in the second term has been set to $n$ with $n>1$. In a flux-conserving scattering which is usually the case in quantum mechanics, the power is $n=1$. This will not be the case for the system under consideration: the D3-observer will measure flux loss since part of the flux will be absorbed into the D5-brane. The absorbed part will form a wave-function that is represented by the third term. The profiling function $\beta(\mathbf{r})$ should be sharply peaked at the three dimensional origin $\mathbf{r}=0$. The third term will contribute to the large$R_{0}$ scattering only through making $n$ higher than one. From the setup, the following property of the cross section can be deduced: a cross section in general reveals certain properties of the target. It is based on what/how much comes out of what has entered. The flux loss in the present setup indicates that there is decay in the wave function. The (differential) cross section will depend on where the measurement is being made. In particular, it will be a decreasing function of $R_{0}$. There will be more discussions using (2) near the end.

There is a large number of literature on scattering that involves D-branes, [32, 33, 34], to name a few. We consider a complementary system of D3/D5. One difference of this setup is that the observer is confined within the D3-brane. The precise supergravity solution has not been obtained. However, there is a configuration made out of intersecting branes $[35,36,37,38,39,40,41]$ that is close.

\footnotetext{
${ }^{3}$ It is an "average distance" from the black hole and the location of the string.

${ }^{4}$ One may pause and wonder whether such a large distance expansion can properly capture the black hole information physics. With regard to this issue, we focus on whether there is a Hawking radiation. If there is, it must be also observed from far away from the black hole. The large distance expansion must be adequate for that.
} 
As stated in the introduction, two geometries, one from the D5 brane and the other from the D3 brane, have different meanings. This is the rationale for different treatments of the two geometries: we will treat the D5-geometry as a potential whereas the D3-geometry will be dealt with as the loop effects of the scattering strings. Since we are only concerned with the tree level scattering, we disregard the D3-geometry curved terms.

The D5-brane that we consider is given by ${ }^{5}$

$$
\begin{aligned}
e^{-\phi} & =H^{1 / 2} \\
d s^{2} & =H^{-\frac{1}{4}}\left(d X^{m}\right)^{2}+H^{\frac{3}{4}}\left(d X^{\mu}\right)^{2} \\
\mathcal{F}_{t y^{1} \cdots y^{5} \mu} & =-2 Q H^{-2} \frac{X^{\mu}}{r^{4}} \\
H & =1+\frac{Q}{r^{2}}, \quad Q=g N \alpha^{\prime}
\end{aligned}
$$

The coordinate $X^{m}$ spans the six Euclidean space and $X^{\mu}$ the four dimensional spacetime. For the scattering amplitude, our proposal amounts to computing an open string two-point function with insertion of a potential vertex operator, ${ }^{6}$

$$
<V\left(x_{1}, k_{1}\right) U_{\text {pot }}(y) V\left(x_{2}, k_{2}\right)>
$$

The locations should be determined based on the physics of scattering. A natural choice is $x_{1}=\infty, x_{2}=0, y=1$. The precise form of $U_{p o t}$ is determined by considering the non-linear model of the D5-brane geometry. In the leading order in $r$, there are only two terms

$$
-\frac{1}{2}\left(\partial_{i} X^{m} \partial^{i} X^{n} \eta_{m n} H^{-\frac{1}{4}}+\partial_{i} X^{u} \partial^{i} X^{v} \eta_{u v} H^{\frac{3}{4}}\right)
$$

The leading term ${ }^{7}$ in the $R_{0}$-expansion of this gives

$$
U_{p o t} \equiv\left(\frac{1}{8} \frac{Q}{R_{0}^{2}} \partial_{i} X^{m} \partial^{i} X^{n} \eta_{m n}-\frac{3}{8} \frac{Q}{R_{0}^{2}} \partial_{i} X^{u} \partial^{i} X^{v} \eta_{u v}\right)
$$

where $Q$ is a constant in (3). $R_{0}$ has appeared as a result of shifting $\mathrm{X}^{m}$ according to (1) and carrying out the large- $R_{0}$ expansion. Let's consider the vector boson vertex operator on the D3-brane,

$$
V_{v}(\zeta, k)=\zeta^{u}\left(\dot{X}^{u}-R^{u v} k^{v}\right) e^{i k \cdot X}
$$

\footnotetext{
${ }^{5}$ The symbol, $r$, is transverse to the D5-brane. It is not to be confused with $r$ of the previous works which is transverse to the D3-brane. For the "average" location, we are using $R_{0}$ instead of $r_{0}$. Since the roles of the two geometries are very different, so are the roles of $r_{0}$ and $R_{0}$.

${ }^{6}$ As far as we can see, this formula is pointed to by an analogy with the standard quantum mechanical treatment of scattering. If preferred, it may be taken as part of our proposal.

${ }^{7}$ The non-linear sigma model of a D5 contains many terms including the parts coming from the Ramond-Ramond fields. They are all sub-leading in the large $R_{0}$-expansion.
} 
Including the polarization vectors, $\left(\zeta^{1}, \zeta^{2}\right)$, and noting that the first term in (6) do not contribute due to dimensional regularization, the two-point correlator becomes

$$
\begin{aligned}
& \quad<V_{v}\left(x_{1}, \zeta^{1}, k^{1}\right) U_{p o t}(1) V_{v}\left(0, \zeta^{2}, k^{2}\right)> \\
& =\quad<V_{v}\left(x_{1}, \zeta^{1}, k^{1}\right)\left[-\frac{3}{8} \frac{Q}{R_{0}^{2}} \partial_{i} X^{u} \partial_{j} X^{v} \eta_{u v}\right] V_{v}\left(0, \zeta^{2}, k^{2}\right)> \\
& \equiv \quad \delta^{4}\left(k_{1}+k_{2}\right) I
\end{aligned}
$$

The fermionic terms in $V_{v}\left(x_{1}, \zeta^{1}, k^{1}\right), V_{v}\left(0, \zeta^{2}, k^{2}\right)$ do not contribute for the same reason. Choosing $x_{1}=\infty, x_{2}=0, y=1$ and taking the string measure into account, one gets, after some algebra which is a simple version of computations in [24, 25],

$$
I=-\frac{3 Q}{4 R_{0}^{2}} \zeta_{1} \cdot \zeta_{2}
$$

The result implies that the cross section decreases as $\frac{1}{R_{0}^{2}}$ after the spherical shell surface integration taken into account. In particular, it vanishes at infinity. Physically this means that there is a total absorption of the flux into the D5 brane.

We now ponder on the ramifications of the result (9) and possible future directions. Although we have considered a D3/D5-brane system, qualitative features will be shared by many other configurations. Generically the wave function will display a decaying behavior due to the inverse field potential. At infinity the cross section vanishes. The outgoing wave does not reach infinity. This feature does not change even for the known extremal D-5 brane solution. Still the leading behavior is $\frac{1}{R_{0}^{2}}$ as we discuss below. The result then implies that Hawking radiation does not occur since it would require a non-decaying wave function as necessary condition. Although we have investigated an extremal case, it does not seem that near-extremal cases will be different in this respect. We may consider what would be a near extremal D5 configuration by first considering a near extremal D1/D5 system and setting the D1-brane charge to zero. To get a non-decaying wave function, therefore Hawking radiation, the leading power of the large- $R_{0}$ expansion of the potential must be $\frac{1}{R_{0}}$. However, the non-extremality does not bring that feature. It is likely that the desired power will come only when there is a spherical symmetry not only for the potential but also for the scattering states. Such symmetry will be possessed by a scalar vertex operator of a closed string, say, dilaton. In that case, Hawking radiation can be realized. This case will not lead to the information paradox either: an observer who has access to a closed string will have access to the detailed physics of the the D5-brane. The observer should live in the bulk. Therefore, it will be understood what is causing the apparent non-unitary evolution.

As just discussed, generically Hawking radiation is not realized. But with a special design, it may be possible. Let us suppose that we have such a setup and scrutinize 
the open string case. This will help us to develop further intuition on the hair and bring out the tasks involved for quantitative understanding of the information "loss". We again resort to the non-relativistic quantum mechanical picture, (2), but now with $n=1$,

$$
e^{i k \cdot x}+\frac{e^{i k r}}{r} f\left(k^{\prime}, k\right)+\beta(\mathbf{r}) \psi_{D 5}
$$

The third part is the wave function of the D5-brane. It does not contribute to the large- $R_{0}$ scattering. It is a product of a four dimensional wave-function, $\beta(\mathbf{r})$, and a wave function in the D5-brane, $\psi_{D 5}$. The wave-function, $\beta(\mathbf{r})$, should be viewed as an invisible part of the $4 \mathrm{D}$ wave-function. The specific question to study is, "is it possible to change the first piece and let the change absorbed by the third piece while keeping the second piece the same?" That would mean that what comes out may be independent of what has entered other than through the mass (or the energy) as we will argue now. Writing the ten dimensional Hamiltonian as a sum of the D3-brane Hamiltonian, $H_{4}$ and the transverse one, $H_{6}$, schematically the Schrodinger equation reads

$$
\left(H_{4}+H_{6}\right)\left[e^{i k \cdot x}+\frac{e^{i k r}}{r} f\left(k^{\prime}, k\right)+\beta(\mathbf{r}) \psi_{D 5}\right]=E\left[e^{i k \cdot x}+\frac{e^{i k r}}{r} f\left(k^{\prime}, k\right)+\beta(\mathbf{r}) \psi_{D 5}\right]
$$

where $H_{4} \beta(\mathbf{r})=e_{4} \beta(\mathbf{r})$ and $\beta(\mathbf{r}) \rightarrow 0$ when $r \rightarrow \infty$. We also assume that $H_{6}$ is purely kinetic. For $r \rightarrow \infty$

$$
H_{4}\left[e^{i k \cdot x}+\frac{e^{i k r}}{r} f\left(k^{\prime}, k\right)\right] \sim E\left[e^{i k \cdot x}+\frac{e^{i k r}}{r} f\left(k^{\prime}, k\right)\right]
$$

For $r \sim 0$, the $\beta(\mathbf{r})$-containing terms will be dominating, ${ }^{8}$

$$
\begin{aligned}
& \psi_{D 5} H_{4} \beta(\mathbf{r})+\beta(\mathbf{r}) H_{6}\left[\psi_{D 5}\right] \sim E\left[\beta(\mathbf{r}) \psi_{D 5}\right] \\
\Rightarrow \quad & H_{6} \psi_{D 5} \sim\left(E-e_{4}\right) \psi_{D 5}
\end{aligned}
$$

The above two equations, (12) and (13), achieve the goal: the change in the 4D wave function, i.e., the change in $\beta(\mathbf{r})$ gets absorbed by the change in $\psi_{D 5}$, therefore not affecting the radially outgoing wave function. Let us set

$$
M_{D 5}=E-e_{4}
$$

where $M_{D 5}$ would be the mass or the energy of the D5-brane. It shows that what comes out is independent of what has entered other than through the mass of the D5brane. So the D3-brane observer will measure the information loss while 10D physics still preserves the unitary evolution of states.

\footnotetext{
${ }^{8}$ Remember that the $\frac{1}{r}$-behavior of the second term is only asymptotically valid.
} 
One may pursue the following future directions: the current setup may be useful to understand the black hole entropy at the full vertex operator level. One direction is along a near-extremal D5 case. The near-extremal brane geometry should presumably be associated with the excitations of massive open strings on the D5-brane. They must be the hair. It will be interesting to compute the entropy of the massive open string states and compare it with the Bekenstein-Hawking entropy. It will also be interesting to study scattering of a scalar state of a closed string. That will make a connection with existing literature.

We end by posing a bold question. Based on the discussion so far, the information paradox does not seem to occur in string theory, regardless of whether there is Hawking radiation or not. However, it occurs in theories based on four dimensions: should the black hole information paradox based on four dimensional models be taken as an indication that our world has more dimensions than four?

\section{Acknowledgements:}

I acknowledge the KITP scholar program of UCSB. This research was supported in part by the National Science Foundation under Grant No. PHY05-51164. I thank Bumhoon Lee for his hospitality during my stay at CQUeST (Sogang university) where the work was completed. This work was supported in part by Korea Science and Engineering foundation through CQUeST with grant number R11-2005-021.

\section{References}

[1] A. Strominger and C. Vafa, "Microscopic Origin of the Bekenstein-Hawking Entropy," Phys. Lett. B 379, 99 (1996) [arXiv:hep-th/9601029].

[2] S. S. Gubser, I. R. Klebanov and A. W. Peet, "Entropy and Temperature of Black 3-Branes," Phys. Rev. D 54, 3915 (1996) [arXiv:hep-th/9602135].

[3] K. Behrndt, G. Lopes Cardoso, B. de Wit, D. Lust, T. Mohaupt and W. A. Sabra, "Higher-Order Black-Hole Solutions in N=2 Supergravity and Calabi-Yau String Backgrounds," Phys. Lett. B 429 (1998) 289 [arXiv:hep-th/9801081].

[4] A. Sen, "How does a fundamental string stretch its horizon?," JHEP 0505, 059 (2005) [arXiv:hep-th/0411255].

[5] A. Dabholkar, R. Kallosh and A. Maloney, "A stringy cloak for a classical singularity," JHEP 0412 (2004) 059 [arXiv:hep-th/0410076]. 
[6] Y. S. Myung, Y. W. Kim and Y. J. Park, "Entropy of an extremal regular black hole," Phys. Lett. B 659, 832 (2008) [arXiv:0705.2478 [gr-qc]].

[7] S. R. Wadia, "Lectures on the microscopic modeling of the 5-dim black hole of IIB string theory and the D1/D5 system," arXiv:hep-th/0006190.

[8] A. W. Peet, "TASI lectures on black holes in string theory," arXiv:hep-th/0008241.

[9] J. Plochinski, "Dirichlet-Branes and Ramond-Ramond Charges," Phys. Rev. Lett. 75, 4724 (1995) [arXiv:hep-th/9510017];

[10] J. Polchinski, "Lectures on D-branes," arXiv:hep-th/9611050.

[11] J. Plochinski, String theory vol 1,2, Cambridge

[12] C. V. Johnson, "D-brane primer," arXiv:hep-th/0007170.

[13] N. Banerjee, I. Mandal and A. Sen, "Black Hole Hair Removal," arXiv:0901.0359 [hep-th].

[14] S. D. Mathur, "Fuzzballs and the information paradox: a summary and conjectures," arXiv:0810.4525 [hep-th]

[15] S. D. Mathur, "What Exactly is the Information Paradox?," Lect. Notes Phys. 769, 3 (2009) [arXiv:0803.2030 [hep-th]].

[16] N. Iizuka, T. Okuda and J. Polchinski, "Matrix Models for the Black Hole Information Paradox," arXiv:0808.0530 [hep-th].

[17] G. Horowitz, A. Lawrence and E. Silverstein, "Insightful D-branes," arXiv:0904.3922 [hep-th].

[18] S. Chandrasekhar, The mathematical theory of black holes, Oxford University Press

[19] S. R. Das, G. W. Gibbons and S. D. Mathur, "Universality of low energy absorption cross sections for black holes," Phys. Rev. Lett. 78, 417 (1997) [arXiv:hepth/9609052].

[20] R. Emparan, "Absorption of scalars by extended objects," Nucl. Phys. B 516, 297 (1998) [arXiv:hep-th/9706204].

[21] E. Jung and D. K. Park, "Effect of scalar mass in the absorption and emission spectra of Schwarzschild black hole," Class. Quant. Grav. 21, 3717 (2004) [arXiv:hepth/0403251].

[22] I. Y. Park, "Scattering on D3-branes," Phys. Lett. B 660, 583 (2008) [arXiv:0708.3452 [hep-th]]. 
[23] I. Y. Park, "One loop scattering on D-branes," Eur. Phys. J. C 62: 783 (2009), arXiv:0801.0218 [hep-th]

[24] I. Y. Park, "Open string engineering of D-brane geometry," JHEP 0808, 026 (2008) [arXiv:0806.3330 [hep-th]].

[25] I. Y. Park, "Geometric counter-vertex for open string scattering on D-branes," arXiv:0902.1279 [hep-th].

[26] M. Cvetic, H. Lu, C. N. Pope and K. S. Stelle, "T-duality in the Green-Schwarz formalism, and the massless/massive IIA duality map," Nucl. Phys. B 573, 149 (2000) [arXiv:hep-th/9907202].

[27] V. Sahakian, "Closed strings in Ramond-Ramond backgrounds," JHEP 0404, 026 (2004) [arXiv:hep-th/0402037].

[28] S. Mizoguchi, T. Mogami and Y. Satoh, "Penrose limits and Green-Schwarz strings," Class. Quant. Grav. 20, 1489 (2003) [arXiv:hep-th/0209043].

[29] W. Fischler and L. Susskind, "Dilaton Tadpoles, String Condensates And Scale Invariance," Phys. Lett. B 171, 383 (1986).

[30] I. Y. Park, Work in progress

[31] M. B. Green, J. H. Schwarz and E. witten, Superstring theory, vol1, Springer

[32] A. Hashimoto and I. R. Klebanov, "Scattering of strings from D-branes," Nucl. Phys. Proc. Suppl. 55B, 118 (1997) [arXiv:hep-th/9611214].

[33] M. R. Garousi and R. C. Myers, "Superstring Scattering from D-Branes," Nucl. Phys. 475 (1996) 193-224, hep-th/9603194

[34] V. Balasubramanian and I. R. Klebanov, "Some Aspects of Massive World-Brane Dynamics," hep-th/9605174

[35] R. R. Khuri, "Remark On String Solitons," Phys. Rev. D 48 (1993) 2947 [arXiv:hep-th/9305143].

[36] J. P. Gauntlett, D. A. Kastor and J. H. Traschen, "Overlapping Branes in MTheory," Nucl. Phys. B 478 (1996) 544 [arXiv:hep-th/9604179].

[37] R. Argurio, "Intersection rules and open branes," arXiv:hep-th/9712170.

[38] N. Ohta, "Intersection rules for non-extreme p-branes," Phys. Lett. B 403 (1997) 218 [arXiv:hep-th/9702164].

[39] J. D. Edelstein, L. Tataru and R. Tatar, "Rules for localized overlappings and intersections of p-branes," JHEP 9806, 003 (1998) [arXiv:hep-th/9801049]. 
[40] J. D. Edelstein and J. Mas, "Localized intersections of non-extremal p-branes and S-branes," JHEP 0406, 015 (2004) [arXiv:hep-th/0403179].

[41] K. S. Cha, B. H. Lee and H. S. Yang, "Intersecting D-branes in type IIB plane wave background," Phys. Rev. D 68, 106004 (2003) [arXiv:hep-th/0307146]. 